\title{
Bioinformation
}

\author{
www.bioinformation.net
}

Hypothesis

\section{Analysis and modeling of mycolyl-transferases in the CMN group}

\author{
Hemalatha Golaconda Ramulu, Swathi Adindla, and Lalitha Guruprasad* \\ School of Chemistry, University of Hyderabad, Hyderabad -500046, India; \\ Lalitha Guruprasad* - Email: lgpsc@uohyd.ernet.in; Phone: +91 40 23134820; Fax: +91 40 23012460; * Corresponding author \\ received May 10, 2006; revised June 14, 2006; accepted June 14, 2006; published online June 18, 2006
}

\begin{abstract}
:
Mycolyl-transferases are a family of proteins that are specifically present in the CMN (Corynebacterium, Mycobacterium and Nocardia) genera and are responsible for the synthesis of cell wall components. We modeled the three-dimensional structures of mycolyltransfersases from Corynebacterium and Nocardia using homology modeling methods based on the crystal structures of mycolyltransferases from $M$. tuberculosis. Comparison of the models revealed significant differences in their substrate binding site. Some mycolyl-transferases identified by the following Gene Ids: Nfa25110, Nfa45560, Nfa7210, Nfa38260, Nfa32420, Nfa23770, Nfa43800, Nfa30260, Dip0365, Ncgl0987, Ce1488, Ncg10885, Ce0984, Ncgl2101, Ncgl0336, Ce0356 are associated with a relatively larger substrate binding site and amino acid residue mutations (D40N, R43D/G, S236N/A) are likely to affect binding to trehalose.
\end{abstract}

Key words: CMN, Mycobacterium; Corynebacterium; Nocardia; mycolyl-transferases; homology modeling

Background: The CMN group constitutes the organisms of the genera Corynebacterium, Mycobacterium and Nocardia, which are grouped together on the basis of factors that include complex cell wall components, presence/type of mycolic acids, adjuvant activity, presence of cord factor, sulfo-lipids, iron-chelating compounds, polyphosphate, and serological cross-reactivity. The cell walls of the organisms that belong to the CMN group consists of interconnected peptidoglycan and polysaccharide-mycolate complex and are characterized by the presence of mycolic acid on their surface. [1] Mycolic acids are long chain fatty acids that form a part of the unique cell envelope, responsible for the pathogenesis and survival of the organism inside the host. The mycolic acids are named according to the individual genus from which they are isolated; i.e., corynomycolic acids from Corynebacterium comprising 22-36 carbons, mycolic/eumycolic acids from Mycobacterium comprising $\sim 60-90$ carbons and nocardiomycolic acids from Nocardia comprising 40-60 carbons. [2-4]

In $M$. tuberculosis, the mycolyl-transferases are also termed antigen 85 or Ag85 complex enzymes. [5] These correspond to three secreted proteins; Ag85A (Gene Id: Rv3804), Ag85B (Gene Id: Rv1886) and Ag85C (Gene Id: Rv0129). These proteins comprise a signal peptide at the $\mathrm{N}$-terminus followed by a carboxylesterase domain. It has been demonstrated that Ag85 enzymes catalyze the transfer of mycolyl residue from one molecule of $\alpha, \alpha^{\prime}-$ TMM (trehalose monomycolate) to another leading to the formation of $\alpha, \alpha^{\prime}-$ TDM (trehalose dimycolate) and hence these enzymes are termed mycolyl-transferases. [6] Also, in Corynebacterium and Nocardia, orthologous proteins synthesize TDCM (trehalose dicorynomycolate) and TDNM (trehalose dinocardiomycolate), respectively. Further, this family of enzymes is specific only to the CMN group of organisms because of their unique cell envelope. Mycolyl-transferases are also termed fibronectin-binding proteins, since they are involved in binding to fibronectin and entry of the organism into host cells. $[7,8]$ Hence, it is important to understand the structure and function of the proteins responsible for the synthesis of cell wall components in CMN.

The structures of Ag85A (PDB Ids: 1SFR) [9], Ag85B (PDB Ids: 1F0N, 1F0P) [10] and Ag85C (PDB Ids: 1DQZ, 1DQY, 1VA5) [11] were determined for both native and substrate bound forms. The structure corresponds to a $\alpha / \beta$ hydrolase fold and the catalytic triad responsible for the mycolyl-transferase activity comprise the amino acid residues S126, E230 and H262 (numbering is according to PDB Id: 1F0P). The structural comparison of the three mycolyl-transferases (PDB Ids: 1SFR, 1F0P, 1DQZ) revealed that the active sites are virtually identical indicating that these share a common function. [9] However, in contrast to the high level of similarity within the substrate-binding site and the active site, it was observed that the surface residues disparate from the active site are quite variable indicating that all three Ag85 enzymes in $M$. tuberculosis are needed to evade the host immune system. The genome sequencing of $M$. tuberculosis [12], C. glutamicum [13], C. efficiens [14], C. diphtheria [15] and Nocardia farcinica [16] is completed. The M. tuberculosis comprising 3,986 genes is the causative agent of tuberculosis that causes 3 million deaths worldwide. The $C$. glutamicum comprising 3,002 genes is a soil bacterium and widely used by the industry in the production of amino acids. The $C$. efficiens comprising 3,069 genes is a non-pathogenic bacterium. The $C$. diphtheria comprising 2,320 genes is the causative agent of diphtheria. The genome of $N$. farcinica comprising 5,674 genes is the causative agent of nocardiosis, affecting the lung, central nervous system and cutaneous tissues of humans and animals.

In our earlier work [17], we identified mycolyltransferases in C. glutamicum and C. efficiens genomes and modeled their three dimensional structures. We reported the relative binding of corynomycolyl-transferases towards trehalose. Our findings are in accordance with the experimental data $[18,19]$ that reported the gene deletion mutation studies and measured the concentration of TMCM / TDCM. The genomes of $N$. farcincia, a representative species from Nocardia and $C$. diphtheria were also subsequently sequenced and we now have complete data available in the public databases on all mycolyl-transferases from species that belong to the CMN group. Therefore we have carried out sequence analysis corresponding to all mycolyl-transferases and modeled the structures of Nocardia and C. diphtheria and compared their substrate binding sites. Such comparative analysis is relevant in 


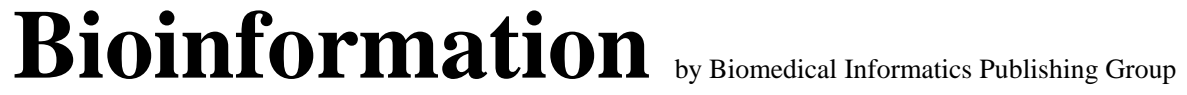

\author{
www.bioinformation.net
}

Hypothesis

situations when the structural information for proteins from only one structure, function and nature of the substrate binding sites for organism is available and useful inferences can be made about the related members from other organisms.

Table 1: Mycolyl-transferases in CMN group

\begin{tabular}{|c|c|c|c|c|c|}
\hline Gene Id & GeneBank Id & Source & Protein Length & $\%$ similarity & $\begin{array}{c}\text { BLASTP } \\
\text { E-value }\end{array}$ \\
\hline Rv1886c & GI:15609023 & M. tuberculosis & 325 & 100 & $9 e-173$ \\
\hline Rv3804c & GI:15610940 & M. tuberculosis & 338 & 90 & $1 e-146$ \\
\hline Rv0129c & GI:57116693 & M. tuberculosis & 340 & 81 & 3e-123 \\
\hline Rv3803c & GI:57117159 & M. tuberculosis & 299 & 52 & $2 e-50$ \\
\hline Nfa1830 & GI:54022147 & N. farcinica & 345 & 53 & $5 e-48$ \\
\hline Nfa1810 & GI:54022145 & N. farcinica & 347 & 51 & $2 \mathrm{e}-47$ \\
\hline Nfa1820 & GI:54022146 & N. farcinica & 353 & 48 & $1 e-45$ \\
\hline NCgl2777 & GI:19554065 & C. glutamicum & 657 & 50 & $2 \mathrm{e}-44$ \\
\hline Ce2709 & GI:25029265 & C. efficiens & 669 & 52 & $5 e-44$ \\
\hline Nfa1840 & GI:54022148 & N. farcinica & 624 & 50 & $1 e-40$ \\
\hline NCgl2779 & GI:19554067 & C. glutamicum & 341 & 50 & $2 e-38$ \\
\hline Dip2193 & GI:38234734 & C. diphtheriae & 638 & 49 & $3 e-38$ \\
\hline Ce2710 & GI:25029266 & C. efficiens & 360 & 51 & $9 e-37$ \\
\hline Dip2194 & GI:38234735 & C. diphtheriae & 338 & 49 & $7 e-35$ \\
\hline Nfa5610 & GI:54022528 & $N$. farcinica & 319 & 48 & $2 e-33$ \\
\hline Nfa30260 & GI:54024995 & N. farcinica & 341 & 45 & $8 e-28$ \\
\hline Nfa32420 & GI:54025211 & N. farcinica & 351 & 44 & $9 e-27$ \\
\hline Nfa38260 & GI:54025796 & N. farcinica & 353 & 42 & $2 \mathrm{e}-26$ \\
\hline Nfa7210 & GI:54022688 & N. farcinica & 340 & 42 & $4 e-26$ \\
\hline Ncgl0987 & GI:19552252 & C. glutamicum & 411 & 45 & $8 e-26$ \\
\hline Nfa25110 & GI:54024480 & N. farcinica & 311 & 45 & $5 e-25$ \\
\hline Ce1488 & GI:25028044 & C. efficiens & 390 & 43 & $9 e-24$ \\
\hline Dip0365 & GI:38232981 & C. diphtheriae & 355 & 43 & $1 \mathrm{e}-23$ \\
\hline Nfa45560 & GI:54026529 & N. farcinica & 324 & 44 & $4 e-23$ \\
\hline Ncgl0885 & GI:19552148 & C. glutamicum & 483 & 43 & $5 e-23$ \\
\hline Ncgl2101 & GI:19553383 & C. glutamicum & 483 & 43 & $8 e-23$ \\
\hline Nfa23770 & GI:54024346 & N. farcinica & 339 & 42 & $4 e-22$ \\
\hline Nfa43800 & GI:54026351 & N. farcinica & 337 & 43 & $9 e-22$ \\
\hline Dip2339 & GI:38234873 & C. diphtheriae & 406 & 44 & $3 e-20$ \\
\hline Ce0356 & GI:25026912 & C. efficiens & 381 & 41 & $5 e-20$ \\
\hline Ce0984 & GI:25027540 & C. efficiens & 484 & 42 & $1 \mathrm{e}-19$ \\
\hline Ncgl0336 & GI:19551592 & C. glutamicum & 365 & 42 & $8 e-18$ \\
\hline
\end{tabular}

Methodology:

Sequence data: The amino acid sequences corresponding to mycolyl-transferases from M. tuberculosis; Ag85A, Ag85B and Ag85C were obtained from the EBI (European Bioinformatics Institute) [20] and are represented by the following Ids; GI: 15610940, GI: 15609023, GI: 57116693, respectively as shown in Table 1.

Database searching: The homologous proteins were identified for the Mycobacterium, Corynebacterium, and $N$. farcinica using BLASTP [21] with the Ag85B as the query sequence against GenBank release 153 [22]. The BLOSUM62 matrices were used and the results were sorted using E-value (expected value) with the gap costs set to existence at 11 and extension at 1 .

Multiple sequence analysis: Thirty-one mycolyl-transferase sequences were aligned using the CLUSTALW program [23] available at EBI. A penalty of 10 for gap opening, 0.05 for gap extension and 8 for gap separation (default parameters) was assigned for the alignment and shown in Figure 1.

Homology modeling: The three-dimensional models were constructed using MODELER [24] available in InsightII (Accelrys ISSN 0973-2063

Bioinformation 1(5): 161-169 (2006)
Inc., USA). The structures of Ag85A (PDB Id: 1SFR), Ag85B (PDB Id: 1F0N) and Ag85C (PDB Ids: 1DQZ) were used as templates for modeling. MODELER is an automated comparative modeling program designed to find the most probable structure of a protein sequence, given its alignment with related structures. The model is obtained by the optimal satisfaction of spatial restraints derived from the alignment and is expressed as probability density function for the features restrained. The optimization procedure is a variable target function method that applies conjugate gradients algorithm to position all non-hydrogen atoms. [25] In all seventeen homology models were constructed for the mycolyl-transferases from $N$. farcincia and $C$. diphtheria species.

Model evaluation: The models were evaluated using PROCHECK. [26] The RMSD (root mean square deviation) values corresponding to topologically equivalent residues between the models and corresponding crystal structures obtained via structural superposition were derived using programs in InsightII (Accelrys Inc., USA) 162 
Table 2: 'Insertion loop’ amino acid sequence, disulphide bridges and substrate binding pockets in CMN mycolyl-transferases

\begin{tabular}{|c|c|c|c|c|c|c|c|c|c|c|c|c|c|c|c|c|c|}
\hline \multirow{2}{*}{$\begin{array}{l}\text { Protein } \\
\text { 1F0P }\end{array}$} & \multirow{2}{*}{$\begin{array}{l}\text { 'Insertion loop' amino acid } \\
\text { sequence }\end{array}$} & \multirow{2}{*}{$\begin{array}{l}\begin{array}{l}\text { Disulphide } \\
\text { bridge }\end{array} \\
\text { Cys } 87- \\
\text { Cys } 92\end{array}$} & \multicolumn{7}{|c|}{ Trehalose 1151 binding residues } & \multicolumn{8}{|c|}{ Trehalose 1152 binding residues } \\
\hline & & & $40 \mathrm{D}$ & $43 \mathrm{R}$ & $126 \mathrm{~S}$ & $223 \mathrm{~N}$ & $262 \mathrm{H}$ & $263 \mathrm{~S}$ & $264 W$ & $154 \mathrm{D}$ & $157 \mathrm{Q}$ & $159 \mathrm{M}$ & $231 \mathrm{~N}$ & $232 \mathrm{~F}$ & $235 \mathrm{~S}$ & $236 \mathrm{~S}$ & $239 \mathrm{~K}$ \\
\hline Rv0129 & & & 38D & $41 \mathrm{R}$ & $124 \mathrm{~S}$ & $221 \mathrm{~N}$ & $260 \mathrm{H}$ & $261 \mathrm{~S}$ & $262 \mathrm{~W}$ & $152 \mathrm{~N}$ & $155 \mathrm{E}$ & $157 \mathrm{~W}$ & $229 \mathrm{G}$ & $230 \mathrm{~L}$ & $233 R$ & $234 \mathrm{~T}$ & $237 \mathrm{~T}$ \\
\hline Rv3804 & & $\begin{array}{l}\text { Cys } 87- \\
\text { Cys } 92\end{array}$ & $40 \mathrm{D}$ & $43 R$ & $126 \mathrm{~S}$ & $223 \mathrm{~N}$ & $262 \mathrm{H}$ & $263 \mathrm{~S}$ & $264 \mathrm{~W}$ & 154D & 157Q & $159 \mathrm{M}$ & $231 \mathrm{G}$ & $232 \mathrm{~F}$ & $235 \mathrm{~T}$ & $236 \mathrm{~S}$ & $239 \mathrm{~K}$ \\
\hline Ncgl2777 & AIGPA & & $40 \mathrm{D}$ & $43 \mathrm{R}$ & $121 \mathrm{~S}$ & $216 \mathrm{G}$ & $261 \mathrm{H}$ & $262 \mathrm{~S}$ & 263W & 149D & $152 \mathrm{~S}$ & 154G & $231 \mathrm{~V}$ & 232I & $235 \mathrm{M}$ & $236 \mathrm{~T}$ & 239T \\
\hline Ce2709 & ATGPA & & 40D & $43 \mathrm{R}$ & $121 \mathrm{~S}$ & $215 \mathrm{G}$ & $261 \mathrm{H}$ & $262 \mathrm{~A}$ & $263 \mathrm{~W}$ & 149D & $152 \mathrm{~S}$ & $154 \mathrm{G}$ & $231 \mathrm{~L}$ & 232I & $235 \mathrm{M}$ & $236 \mathrm{~T}$ & 239T \\
\hline Ncgl2779 & DH & & $41 \mathrm{D}$ & $44 \mathrm{R}$ & $128 \mathrm{~S}$ & $223 \mathrm{~V}$ & $266 \mathrm{H}$ & $267 \mathrm{G}$ & $268 \mathrm{~W}$ & $156 \mathrm{~N}$ & $159 \mathrm{~A}$ & $161 \mathrm{G}$ & $236 \mathrm{~F}$ & $237 \mathrm{~V}$ & $240 \mathrm{~T}$ & $241 \mathrm{~S}$ & $244 \mathrm{I}$ \\
\hline Ce2710 & DH & & $41 \mathrm{D}$ & $44 \mathrm{R}$ & $128 \mathrm{~S}$ & $223 \mathrm{~T}$ & $266 \mathrm{H}$ & $267 \mathrm{~S}$ & $268 \mathrm{~W}$ & $156 \mathrm{~T}$ & $159 \mathrm{~A}$ & $161 \mathrm{G}$ & $236 \mathrm{~A}$ & $237 \mathrm{~V}$ & $240 \mathrm{~A}$ & $241 \mathrm{~T}$ & $244 \mathrm{~A}$ \\
\hline Ncgl0987 & SEKEPFYN & & $41 \mathrm{D}$ & $44 \mathrm{G}$ & $125 \mathrm{~S}$ & 219D & $267 \mathrm{H}$ & $268 \mathrm{~N}$ & $269 \mathrm{~W}$ & $153 \mathrm{~S}$ & $156 \mathrm{D}$ & $158 \mathrm{I}$ & $240 \mathrm{~S}$ & $241 \mathrm{C}$ & $244 \mathrm{~A}$ & $245 \mathrm{~L}$ & $248 \mathrm{~S}$ \\
\hline Ce1488 & YADEPFYN & & $41 \mathrm{D}$ & $44 \mathrm{G}$ & $125 \mathrm{~S}$ & $219 \mathrm{E}$ & $267 \mathrm{H}$ & $268 \mathrm{~N}$ & $269 \mathrm{~W}$ & $153 \mathrm{~S}$ & 156D & $158 \mathrm{I}$ & $240 \mathrm{~S}$ & $241 \mathrm{C}$ & $244 \mathrm{~A}$ & $245 \mathrm{~L}$ & $248 \mathrm{~A}$ \\
\hline Ncgl0885 & DNAPIDEDAFKNR & & $41 \mathrm{G}$ & $44 \mathrm{D}$ & $124 \mathrm{~S}$ & - & $272 \mathrm{H}$ & $273 \mathrm{~A}$ & $274 \mathrm{~W}$ & $152 \mathrm{E}$ & $155 \mathrm{~S}$ & $157 \mathrm{M}$ & $241 \mathrm{~A}$ & $242 \mathrm{M}$ & $245 \mathrm{~T}$ & $246 \mathrm{C}$ & $249 \mathrm{~N}$ \\
\hline Ce0984 & ENAPEDEKGLKNR & & $41 \mathrm{G}$ & $44 \mathrm{D}$ & $124 \mathrm{~S}$ & - & $272 \mathrm{H}$ & $273 \mathrm{~A}$ & $274 \mathrm{~W}$ & $152 \mathrm{E}$ & $155 \mathrm{~S}$ & $157 \mathrm{M}$ & $241 \mathrm{~A}$ & $242 \mathrm{~L}$ & $245 \mathrm{~T}$ & $246 \mathrm{C}$ & $249 \mathrm{~N}$ \\
\hline Ncgl2101 & DNAPIDEDAFKNR & & $41 \mathrm{G}$ & $44 \mathrm{D}$ & $124 \mathrm{~S}$ & - & $272 \mathrm{H}$ & $273 \mathrm{~A}$ & $274 \mathrm{~W}$ & $152 \mathrm{E}$ & $155 \mathrm{~S}$ & $157 \mathrm{M}$ & $241 \mathrm{~A}$ & $242 \mathrm{M}$ & $245 \mathrm{~T}$ & $246 \mathrm{C}$ & $249 \mathrm{~N}$ \\
\hline Ncgl0336 & SPRFEGLNQQVQSIAMAET & & $41 \mathrm{~N}$ & $44 \mathrm{D}$ & $124 \mathrm{~S}$ & 218D & $276 \mathrm{H}$ & $277 \mathrm{~S}$ & 278W & $152 \mathrm{~A}$ & $155 \mathrm{~S}$ & 157L & $246 \mathrm{~A}$ & $247 \mathrm{~A}$ & $250 \mathrm{~K}$ & $251 \mathrm{C}$ & 254D \\
\hline Ce0356 & SPRFNGLDQAYLSLAMTET & & $41 \mathrm{~N}$ & $44 \mathrm{D}$ & $124 \mathrm{~S}$ & $218 \mathrm{~N}$ & $276 \mathrm{H}$ & $277 \mathrm{~S}$ & $278 \mathrm{~W}$ & $152 \mathrm{~S}$ & $155 \mathrm{Q}$ & 157L & $246 \mathrm{~A}$ & $247 \mathrm{~A}$ & $250 \mathrm{~K}$ & $251 \mathrm{C}$ & $254 \mathrm{D}$ \\
\hline Nfa1810 & FG & & $40 \mathrm{D}$ & $43 R$ & $153 \mathrm{~S}$ & $249 \mathrm{~N}$ & $291 \mathrm{H}$ & $292 \mathrm{~N}$ & $293 W$ & $181 \mathrm{~N}$ & $184 \mathrm{~A}$ & $186 \mathrm{G}$ & $260 \mathrm{~V}$ & $261 \mathrm{~L}$ & $264 \mathrm{~A}$ & $265 N$ & $268 \mathrm{~A}$ \\
\hline Nfa1820 & FN & & 40D & $43 \mathrm{R}$ & $148 \mathrm{~S}$ & $244 \mathrm{~S}$ & $286 \mathrm{H}$ & $287 \mathrm{~A}$ & $288 \mathrm{~W}$ & $176 \mathrm{~N}$ & $179 \mathrm{~A}$ & $181 \mathrm{G}$ & $255 \mathrm{~A}$ & $256 \mathrm{~L}$ & $259 \mathrm{~A}$ & $260 \mathrm{~N}$ & $263 \mathrm{~A}$ \\
\hline Nfa1830 & SPVGVFN & & 39D & $42 \mathrm{R}$ & $124 \mathrm{~S}$ & $218 \mathrm{~N}$ & $264 \mathrm{H}$ & $265 S$ & 266W & $152 \mathrm{~N}$ & $155 \mathrm{~A}$ & $157 \mathrm{G}$ & $234 \mathrm{~A}$ & $235 \mathrm{~L}$ & $238 \mathrm{~V}$ & $239 \mathrm{~N}$ & $242 \mathrm{~A}$ \\
\hline Nfa1840 & PGVST & & $41 \mathrm{D}$ & $44 \mathrm{R}$ & $122 \mathrm{~S}$ & $217 \mathrm{~S}$ & $263 \mathrm{H}$ & $264 \mathrm{~S}$ & $265 \mathrm{~W}$ & $150 \mathrm{~T}$ & $153 \mathrm{~T}$ & 155G & 233I & $234 \mathrm{~L}$ & $237 \mathrm{~L}$ & $238 \mathrm{~T}$ & $241 \mathrm{~N}$ \\
\hline Nfa25110 & & $\begin{array}{l}\text { Cys 146- } \\
\text { Cys } 227\end{array}$ & $38 \mathrm{~A}$ & $41 \mathrm{G}$ & $120 \mathrm{~S}$ & - & $252 \mathrm{H}$ & $253 \mathrm{~T}$ & $254 \mathrm{~W}$ & $148 \mathrm{~W}$ & $151 \mathrm{D}$ & $153 \mathrm{P}$ & $222 \mathrm{~A}$ & 223I & $226 \mathrm{~T}$ & $227 \mathrm{C}$ & $230 \mathrm{~A}$ \\
\hline Nfa45560 & APGIDGNPLDLVER & $\begin{array}{l}\text { Cys } 146- \\
\text { Cys } 242\end{array}$ & $38 \mathrm{~N}$ & $41 \mathrm{D}$ & $120 \mathrm{~S}$ & $241 \mathrm{~T}$ & $266 \mathrm{H}$ & $267 S$ & $268 W$ & $148 \mathrm{R}$ & $151 \mathrm{D}$ & $153 \mathrm{~A}$ & $237 \mathrm{~T}$ & $238 \mathrm{~V}$ & $241 \mathrm{~A}$ & $242 \mathrm{C}$ & $245 \mathrm{P}$ \\
\hline Nfa7210 & GPYALPGSYGLANQ & $\begin{array}{l}\text { Cys } 149- \\
\text { Cys } 246\end{array}$ & $41 \mathrm{~N}$ & $44 \mathrm{G}$ & $123 \mathrm{~S}$ & $218 \mathrm{~N}$ & $271 \mathrm{H}$ & $272 S$ & $273 W$ & $151 Q$ & $154 \mathrm{D}$ & $156 \mathrm{~V}$ & $241 \mathrm{~A}$ & $242 \mathrm{G}$ & $245 Y$ & $246 C$ & $249 N$ \\
\hline Nfa38260 & GPHAMPGSDGLTNQ & $\begin{array}{l}\text { Cys } 150- \\
\text { Cys } 246\end{array}$ & $41 \mathrm{~A}$ & $44 \mathrm{G}$ & $123 \mathrm{~S}$ & $217 \mathrm{~N}$ & $270 \mathrm{H}$ & $271 S$ & $272 W$ & $151 Q$ & $154 \mathrm{D}$ & $156 \mathrm{~V}$ & $240 \mathrm{~A}$ & $241 \mathrm{G}$ & $244 \mathrm{H}$ & $245 C$ & $248 N$ \\
\hline Nfa32420 & YLNAAPGPMGAVN- & $\begin{array}{l}\text { Cys } 150- \\
\text { Cys } 246\end{array}$ & $41 \mathrm{~N}$ & $44 \mathrm{D}$ & $123 \mathrm{~S}$ & $218 Y$ & $270 \mathrm{H}$ & $271 Y$ & $272 W$ & $151 Q$ & $154 \mathrm{D}$ & $156 \mathrm{~T}$ & $240 \mathrm{~A}$ & $241 \mathrm{~A}$ & $244 \mathrm{Q}$ & $245 C$ & $248 \mathrm{~N}$ \\
\hline $\mathrm{Nfa} 23770$ & NPRLHNDDRQLLNQ & $\begin{array}{l}\text { Cys } 157- \\
\text { Cys } 253\end{array}$ & $41 \mathrm{~N}$ & $44 \mathrm{G}$ & $130 \mathrm{~S}$ & $224 \mathrm{~A}$ & $278 \mathrm{H}$ & $279 S$ & $280 \mathrm{~W}$ & $158 \mathrm{M}$ & $161 \mathrm{D}$ & $163 \mathrm{~L}$ & $247 \mathrm{~S}$ & $248 \mathrm{~V}$ & $241 \mathrm{~L}$ & $252 \mathrm{C}$ & $255 R$ \\
\hline Nfa43800 & AVGGDPMQLGYQ & $\begin{array}{l}\text { Cys } 149- \\
\text { Cys } 243\end{array}$ & $41 \mathrm{~N}$ & $44 \mathrm{~S}$ & $122 \mathrm{~S}$ & - & $267 \mathrm{H}$ & $268 \mathrm{~A}$ & $269 \mathrm{~W}$ & $150 \mathrm{R}$ & 153D & $155 \mathrm{Q}$ & $237 \mathrm{~A}$ & $238 \mathrm{~V}$ & $241 \mathrm{M}$ & $242 \mathrm{C}$ & $245 \mathrm{Q}$ \\
\hline $\mathrm{Nfa} 30260$ & GPGIDADPLALADQ & $\begin{array}{l}\text { Cys } 149- \\
\text { Cys } 245\end{array}$ & $41 \mathrm{~N}$ & $44 \mathrm{~T}$ & $123 \mathrm{~S}$ & $217 \mathrm{Q}$ & $270 \mathrm{H}$ & $271 S$ & $272 W$ & $151 P$ & $154 \mathrm{D}$ & $156 \mathrm{R}$ & $240 \mathrm{~A}$ & $241 \mathrm{~V}$ & 244D & $245 C$ & $248 \mathrm{E}$ \\
\hline Nfa5610 & KPQLAEN & $\begin{array}{l}\text { Cys } 148- \\
\text { Cys } 235\end{array}$ & $41 \mathrm{D}$ & $43 \mathrm{D}$ & $122 \mathrm{~S}$ & $214 \mathrm{~L}$ & $260 \mathrm{H}$ & $261 S$ & $262 W$ & $150 \mathrm{D}$ & $153 \mathrm{~L}$ & $155 \mathrm{~T}$ & $230 \mathrm{~V}$ & $231 \mathrm{G}$ & 234I & $235 \mathrm{C}$ & $238 \mathrm{~A}$ \\
\hline Dip0365 & SPRLAGKDPVTIFATNLIT & & $39 \mathrm{~N}$ & $42 \mathrm{D}$ & $122 \mathrm{~S}$ & $216 \mathrm{~S}$ & $274 \mathrm{H}$ & $275 S$ & $276 \mathrm{~W}$ & $150 \mathrm{~A}$ & $153 \mathrm{~S}$ & $155 \mathrm{~L}$ & $244 \mathrm{~A}$ & 245G & $248 \mathrm{M}$ & $249 \mathrm{C}$ & $252 \mathrm{D}$ \\
\hline Dip2339 & PKEDGPFT & & $41 \mathrm{D}$ & $44 \mathrm{~T}$ & $125 \mathrm{~S}$ & 219G & $269 \mathrm{H}$ & $270 \mathrm{~S}$ & $271 \mathrm{~W}$ & $153 \mathrm{~S}$ & $156 \mathrm{~N}$ & $158 \mathrm{~S}$ & $240 \mathrm{R}$ & $241 \mathrm{C}$ & $244 \mathrm{E}$ & $245 \mathrm{~L}$ & $248 \mathrm{~S}$ \\
\hline Dip2193 & ANKKG & & 40D & $43 \mathrm{R}$ & $121 \mathrm{~S}$ & 215G & $261 \mathrm{H}$ & 262D & $263 W$ & 149D & $152 \mathrm{~S}$ & 154G & $231 \mathrm{~V}$ & 232I & $235 \mathrm{M}$ & $236 \mathrm{~T}$ & 239T \\
\hline Dip2194 & ND & & $41 \mathrm{D}$ & $44 \mathrm{R}$ & $125 \mathrm{~S}$ & $220 \mathrm{Y}$ & $263 \mathrm{H}$ & $264 \mathrm{~N}$ & $265 \mathrm{~W}$ & $153 \mathrm{~S}$ & $156 \mathrm{~V}$ & $158 \mathrm{G}$ & $233 \mathrm{I}$ & $234 \mathrm{~A}$ & $237 \mathrm{~V}$ & $238 \mathrm{~S}$ & 241I \\
\hline
\end{tabular}

The method of Profiles-3D that measures the compatibility of an amino acid sequence to a protein of known three-dimensional structure was used to further assess the model. [27]

Substrate docking: The trehalose substrate was docked into the binding site of all protein models using QUANTA (Accelrys Inc., USA). The enzyme-substrate complex was refined using molecular mechanics (MM) and molecular dynamics (MD) calculations in order to understand their interactions. Hydrogen atoms were added to the structures at $\mathrm{pH} 7.00$ using BIOPOLYMER in Insight II. The parameter 'capping mode off' was chosen so that the protein ends remain uncharged with the $\mathrm{NH} 2$ and $\mathrm{COOH}$ groups. The CVFF (Consistent Valence Force Field) force field was chosen and the

Results and Discussion:

Sequence searches identified four mycolyl-transferases each in $M$. tuberculosis and C. diphtheria, six in C. glutamicum, five in $C$. efficiens, and thirteen in $N$. farcinica. The details of mycolyltransferases analysed and modeled in this work are provided in Table 1. The mycolyl-transferases corresponding to the mycobacteria species; $M$. tuberculosis, $M$. leprae and $M$. bovis are highly similar. Therefore, the mycolyl-transferases from $M$. tuberculosis $\mathrm{H} 37 \mathrm{Rv}$ strain are used in our analysis. Also, $M$. tuberculosis consists a mycolyl-transferase precursor protein MPT51 (Gene Id: Rv3803) that does not possess mycolyl-transferase activity [28] and was also therefore excluded from our analysis. The multiple

ISSN 0973-2063

Bioinformation 1(5): 161-169 (2006)
'Fix' option was used to select the potential atom types, partial charges and formal charges for the protein-substrate complex. The docked complex was subjected to energy minimization using 3000 steps steepest descent followed by conjugate gradient until an energy gradient < 0.01 $\mathrm{kcal} / \mathrm{mol} / \mathrm{A}^{0}$ was achieved. The energy minimized structures were further subjected for MD simulations which were performed in the canonical ensemble (NVT) at $298^{\circ} \mathrm{K}$ using CVFF force field implemented in Discover-3 and equilibrated for 3000 femtoseconds with step size of 1 femtosecond.

sequence alignment of thirty-one mycolyl-transferases is shown in Figure 1. Despite low sequence similarity shared between these proteins, we observed 16 amino acid residues are conserved. These amino acid residues are; L39, W51, P71, D81, W82, W97, F100, G124, S126, S150, D192, G214, E230, G260, H262 and W264. The alignment also indicated some proteins have an insertion sequence of variable length (between 2 and 19 amino acid residues) that precedes the catalytic E230. Further, two $N$. farcinia proteins (Nfa1810 and Nfa1820) comprise a 27 amino acid residue insertion sequence rich in glycine and serine present between the conserved W82 and W97 (see Figure 1). 
Figure 1: Multiple sequence alignment corresponding of CMN mycolyl-transferases. Conserved amino acid residues (*), sites of insertion (inverted triangle).

Nfa7210

Nfa3 8260

$\mathrm{Nfa} 32420$

Nfa2 3770

$\mathrm{Nfa} 43800$

Nfa 30260

Nfa 45560

Nfa2 5110

Nfa5610

Ce0356

Ncgl0336

Dip0365

Ncgl2101

Ncgl 0885

$\mathrm{Ce} 0984$

Ce1488

Ncgl0987

Dip2339

$\mathrm{Ce} 2709$

Ncgl2 277

Dip2193

Nfa1840

Nfa1810

Nfa1820

Nfa1830

$1 F 0 P$

Rv3804C

$\operatorname{Rv} 0129 \mathrm{c}$

Ce2710

Ncgl2 779

Dip2194

Nfa7210

Nfa 38260

Nfa3 2420

Nfa2 3770

$\mathrm{Nfa} 43800$

Nfa 30260

Nfa 45560

Nfa2 5110

Nfa5610

Ce0356

Ncgl0336

Dip0365

Ncgl2101

Ncgl 0885

$\mathrm{Ce} 0984$

$\mathrm{Ce} 1488$

Ncg10987

Dip2339

$\mathrm{Ce} 2709$

Ncgl2777

Dip2193

Nfa1840

Nfa1810

Nfal 820

Nfa1830

$1 F 0 P$

Rv3804C

Rv0129c

Ce2710

Ncgl 2779

Dip2194
IKDDRNLRLYVYSAAMDENVIIDVQRPADASVPRPTLYLLNGAGGGEDDASWVAKSDALK 60 VVDARTVRLRVYSAAMGRVIDIDVQRPADTGAPRPTLYLLAGAGGGEDSASWAKQTSVLE 60 AKEGRTWHLTVYSAAMDTEIAVDVQRPADDSVPAPNLYMLNGLDGGEGTASWAAATHALD 60 GTPARLVDLAVYSPAMQRSIAVKVLRPADTTRPAPTLYLLNGAGGGEDAANWFGQTDAVE 60 PENDRLLDLEIHSPAMDSTTRVLLLRAPDPDRPAPTLYLLNGASGHVDG-SWHDRTDYQR 59 PRSDREVEVIVHSAAMAAEIPIRLLRAADPDRPAPTLYLLNGITGGGDGGNWFDRTGVAA 60 PLGGRQLEVVVHSAAMNRPITLWMS - - HPGPGAPALYLLNAVDGGEDGGPWMNRTDVAA 57 PLAPRVDQVQVYSPSMDAVVSSTVIR - - ADGPAPTLYLLAGAGGGTDGISWWHHTDVRQ 57 ELSPTRSAVFVDSPAMGRVIQVQVLHP - AGGAARP SYYLLDGLDPGVGQSTWTNATDAEA 59 ASGERVKEMWAYSPSMDRDVPLVVITADESAGPRPVIYLLNGGDGGEGNANWIMQTDVID 60 AADERVKEMWAYSPSMDRNVPLVVITADESAGPRPVIYLLNGGDGGEGAANWVMQTDVLD 60 ATGDRVVEMWAHSPSMNRNVPLVVLKAANPG--RPTIYLLNGGDGGEGSANWVMQTKALD 58 VDGDRIROINAYSPSMGRTIPLVWVVPEDNTVPGPTVYALGGGDGGQGGQNWVTRTDLEE 60 VDGDRIRQINAYSPSMGRTIPLVWVVPEDNTVPGPTVYALGGGDGGQGGQNWVTRTDLDE 60 VDGERIRQINAYSP SMERWIPLVWIVPEDTSEPRPTLYALGGGDGGQGSANWITKTDMPE 60 MDGLRLERWTVASPSMQRNVDVQIMRSVDAGAPAPMLYMLDGIGGNRNSSGWINHGQGPK 60 LNGLRLEKWSVASP SMQRNVDVQIMKSAEADSPAPMLYMLDGIGGNKNSSGWINGGEGPK 60 DERFDVDRLF IESPAMRRIVQVQVQHPKDRTTPAPMLYLLDGVTAP - SQSGWLRKGDVQG 59 HVVLSIQSAAMPERPIKVQLLLPRDWYSSPDRDFPEIWALDGLRAIEKQSGWTIETNIEQ 60 HVILTIQSAAMPERPIKVQLLLPRDWYSSPNREFPEIWALDGLRAIEEQSGWTIETNIEQ 60 RVAVYVNTPSMG - QVQVQILLARDWFQDPNRSFPSVWALDGLRATDVENGWTIGTNIEQ 58 RVALWVNSPSMG-APVQVQLLLARDWNAKPEARFPLLIMLDGLRATDDESGWTKDAGAEE 59 SAAFNPDGFDFWVDSDMGPIKSRIFRA-ADGNTNRVVYALDGMRARNDLSGWEIDTEVAR 59 SAAFDPAAFDFWVDSGMGPIKSRILRA-ADGNTNRVVYVLDGMRAPETLNGWEIETDVPA 59 LRAPAGGYEELMVPSVMGP IKVQVQWA-SRG-GDAALYLLDGLRARDDRNAWSFETNAME 58 FSRPGLPVEYLQVPSPSMGRDIKVQFQ-SGGNNSPAVYLLDGLRAQDDYNGWDINTPAFE 59 FSRPGLPVEYLQVPSP SMGRDIKVQFQ-SGGANSPALYLLDGLRAQDDF SGWDINTPAFE 59 FSRPGLPVEYLQVPSASMGRDIKVQFQ-GGG--PHAVYLLDGLRAQDDYNGWDINTPAFE 57 WDGVGYWVQRCDVYSPAMGRNIAVQIQPAQRGGNAGLYLLDGMRATTWSNAWLVDTNAAA 60 WDAVGFWVQRCDVWSPAMGRNIPVQIQPAGRGGNAGLYLLDGMRATEYSNAWLVDTNAAR 60 WDGVAHWVQRCDVFSPAMGRNITVQIQPAQRGGNAALYLLDGARANEIANAWTTDAHVQD 60

\section{$\nabla$}

FLSDKNVNVIQPIGGKWSYYTDWIKDDP FLADKNVNVVQPIGGAWTYYTDWRAPDP - - WLADKPVNVIQPIGGRGSYYTDWLRRDP FFADKHVNVVIPMEGAFSYYTDWERADEGLAE - - - - - - TLGNN 97 FFADKQVNVVIPLGGAGSYFTDWRAEDP - - - - FFAGEQVNVAMPIGGAGSYFADWRARDP FFADKNVNVIVPMGGRASYYTDWVADDP - - - - FFADKNVNVVMPIGGRF SLYTDWQADDP - FFRGKNVNVVLPVGGQASYYTDWQTDDP - - - FYLEKNVNVVIPMEGKF SYYTDWVQENA - - - - FYLEKNVNVVIPMEGKFSYYTDWVEENA- - FYRDKDVNVVIPMAGKF SYYTDWVSEAP - - - LTSDNNINLIMPMLGSFSFYADWAGESE- LTSENNINLIMPMLGSFSFYADWAGESE------ - - - - - - - - - SMG- 91 LMSSNNVHVIMPMLGSHSFYADWVEEND- VFGDENVTVVMPLGAAASMYSDWVEEDP - - - - - - - - - - - - - ALG - 91 VFADENVTVVMPLGAASSMYSDWLEEDP - -

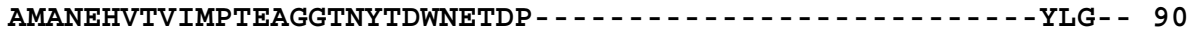

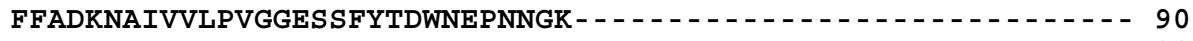
YYADKNAIVVLPVGGESSFYSDWEGPNNGK - $-\ldots-\ldots$ FYSDKNVNVILPVGGQSSFYSDWQQPNNGK FFADKNVTVVLPVGGQSSFYADWMQPNNGR ELTKWNINVVMPVGGMSSFYADWNAPSTILGIGGGSSGSASGSSSGSGALQMFAGGPGKS 119 LLASWNINVVMPVGGMSSFYADWNAP SEFFGIPAGS - - - - -GSSSGSGALNAFTGGPGKS 114 QFKNDNITLVMPVGGQSSFYTDWYAPSNTN- - - - - - - - - - - - GQK 91 WYYQSGLSIVMPVGGQSSFYSDWYSPACGK -

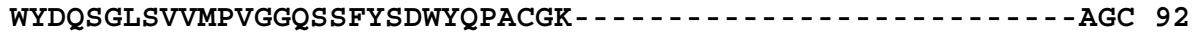

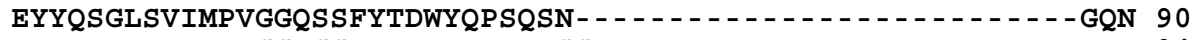
LYAPHNITLVMPVGGAGSFYADWNHPATLSSA LYAPNNITLVMPVGGAGSFYADWNSQASLSSS LFVDHNITLVMPVGGAGSFYTDWVGPAGPQN-_......... 91 
Nfa 7210

Nfa38260

Nfa32420

Nfa2 3770

Nfa 43800

Nfa 30260

Nfa 45560

Nfa2 5110

Nfa5610

$\mathrm{Ce} 0356$

Ncgl 0336

Dip0365

Negl2101

Ncgl 0885

$\mathrm{Ce} 0984$

Ce1488

Ncgl0987

Dip2339

$\mathrm{Ce} 2709$

Ncgl2 777

Dip2193

Nfa1840

Nfa1810

Nfa1820

Nfa1830

1FOP

Rv3804C

Rv0129c

Ce2710

Ncgl 2779

Dip2194

Nfa7210

Nfa 38260

Nfa32420

Nfa2 3770

$\mathrm{Nfa} 43800$

Nfa 30260

Nfa 45560

Nfa2 5110

Nfa5610

Ce0356

Ncgl 0336

Dip0365

Ncgl2101

Ncgl 0885

$\mathrm{Ce} 0984$

Ce1488

Ncgl0987

Dip2339

Ce2709

Ncgl2777

Dip2193

Nfa1840

Nfa1810

Nfa1820

Nfa1830

$1 \mathrm{FOP}$

Rv3804C

Rv0129c

Ce2710

Ncgl2779

Dip2194

\section{$\nabla$}

-RNKWKTFFTEELP - - PLVDGALGTNGINAIAGLSTSGTTVLALPIAKPGLYKAAAAYS 147 -VNKWKTFLTEELP - - - PVIDAALGTNGVNALAGLSMSGTSALQLPIAAPGLYRAVAAYS 147 -MNKWRTFFTEELP - - - PLLDATLRSTGRNALTGLSTSGTSVLQLAEAKPGLWRSVAAYS 147 GRNMWTTFLTEELP - - - PVIDATFGATGANALAGISMAGSSVLDLTIQAPTRYRSVAAYS 154 -RQRWATFLTEELP - - - PLLDEHFHGSGANAVAGVSMSGTSVFQLALAAPGLYRAIGSFS 146 -LQRWASFLTRELP - - PLLDNAFRGTGANAVIGVSMAGTSVFQLALHAPGVYRAIGSFS 147 -RNKWSTFLTAELP - - PLLEQRFGMTGRNAVAGLSMSATSALNLALDAPGRYQAVGAYS 144 -RNRWQTFLTRELP - - AAMTPWLGATGRDAIAGVSMSAASAIDLAIQAGDRYRAVAAYS 144 -RYKWETFLTRELP - - - PIIDAQFAGNGVNGIGGLSMGGNAAYILAARNPHLYTAVAGYS 146 GKQMWETFLVKELP - - - GPLEEELNADGQRAIAGMSMSATTSLLFPQHYPGFYDAAASFS 148 GKQMWETFLVKELP - - GPLEEKLNTDGQRAIAGMSMSATTSLLFPQHF PGFYDAAASFS 148 GKQNWETFLTKELP - - -GPIERHLGASNKRAIAGLSMSATSALVLAEHAQGFYDAAGSFS 146 GAQQWETFLMHELP - - - EPLEAAIGADGQRS IVGMSMSGGSVLNFATHDPNFYSSVGSFS 148 GAQQWETFLMHELP - - EPLEAAIGADGQRS IVGMSMSGGSVLNFATHDPNFYSSVGSFS 148 GKQQWETFLTHELP - - - EPLEAAIGGDGQRSIIGMSMSGGSVVNIASHQPNFYSSVASLS 148 -RIMWETFIVEELA - PLLEAEEELNFNGHRGIGGLSMGATGAVHLANANPDFFDAVIGIS 149 -RIKWETF IVEELA - PLLEAEEELNFNGHRGIGGLSMGATGAVHLANSNPDLFDGVIGIS 149 -RAKWETFLIKELPGVLVQPETKIAYNGKSYIGGLSMGGSAAVRLANLYPEKFVGTFGVS 149 -NYQWETFLTEELA - - PILDKGFRSN-GERAITGISMGGTAAVNIATHNPEMFNFVGSFS 146 -NYQWETFLTQELA- - PILDKGFRSN-TDRAITGISMGGTAAVNIATHHPDMFKFVGSFS 146 -HYKWETFLTNELV - - PVLKNGFRTN-DDRAVVGLSMGGTAAINLAERRPDLFKFVGSFS 144 -NYKWETFLTKELP - - PLLESQWRAT-DVRGMQGLSMGGTAAMFLAGRNPGFVRYAASYS 145 TRYTWETFLTNNLR - -WALRDRLGFNPNRNGVFGLSMGGSAALTLAAYHPDQFSYAGSYS 177 YRYQWETFLTNELR - -WALRDRLGFNPNRNGVFGLSMGGSAALTLAAYHPDQFSFAGSFS 172 TTYKWETFLTQELP - -NFLAG-YGVSKTNNAVAGLSMGGSAALALAAYHRDQFKYAASYS 148 QTYKWETFLTSELP - -QWLSANRAVKPTGSAAIGLSMAGSSAMILAAYHPQQFIYAGSLS 150 QTYKWETFLTSELP - -GWLQANRHVKPTGSAVVGLSMAASSALTLAIYHPQQFVYAGAMS 150 YTYKWETFLTREMP - -AWLQANKGVSPTGNAAVGLSMSGGSALILAAYYPQQFPYAASLS 148 VVYMWETFLTAELP - - AYLEQHFGVARNNNSVAGLSMGGTAALNLAAKHPGQFRQAMSYS 152 VIYMWETFLTQELP - -AYLEQNFGVARNNNSIGGLSMGGTAALNLAAKHPDQFRQAMSWS 152 AIYRWETFLTQELP - -GYLAANFGVSPTNNSIAGLSMGATAAMNLAALHPDQFRQVLSYS 149

GCAQTSDPVGSEFVKLTVETWGGGDTENMWGPPGSEEWVKNDPYVNAEGLRG- - - LELYI 204 GCAQISDPVGHHFV - ATVVAAGHGDVVNMYGPPDDPMWAANDPYVQAERLRG- - - LELFL 203 GCAQIADPTGRQFVKLAVETWAGGDTENMYGPDDSPLWRENDPVVNAEKLRG- - TQLYI 204 GCAMTSDPLGRMFV - TVVISLGGGDPENMWGPTGGDGWREHDPYLQAHRLPP - - - IPMYI 210 GCVRTSDPQGQVMVNAVVASHR-GNPVNMWGPPTDPTWRANDPYLHADRLRG-- -TAIYI 202 GCVPTSDARGRAVVNTVVRAYG-GDPVNLWGPPEDPAWAANDPSLRAAELRD-- -TAVYV 203 GCARTSDPAGRALIYAELAVFG-ANATNMWGGPDSPLWAAHDPVLRAEELRG- - - LAIYV 200 GCPWRADP PGMTLVAAQVLRGG-GNPVNMWGP PGDPGWQSHDAFRNAGALAG- - -KTVYL 200 ACPDTGLATG - - AVMF SIANRG-GNPLNMWGPPGSPAWAEHDPARLAGNLRG- - KTLYL 200 GCASTSQPLPWEYIRLTLDRGN-ATPEQMWGPRGGEVNIYNDALINSDKLRG- - -TDLYI 204 GCAATSSLLPWEYLKLTLDRGN-ATPEQMWGPRGGEYNIYNDALINSDKLRG- - TELYV 204 GCAATSSPLTYHFLRLTLERGG-ATPEQMWGPOGSEVNRYNDALINAERLRG- - - TEVYV 202 GCAETNSWMGRRGIAATAYNGN-VVPEQIFGEVDSDYSRYNDPLLNAAKLEE--QDNLYI 205 GCAETNSWMGRRGIAATAYNGN -VVPEQIFGEVDSDYSRYNDPLLNAAKLEE--QDNLYI 205 GCAETNSWMGRRGVAATVYSGN-ATPTQIFGEVDSDYARYNDPVINAHRLAK - -QDNLYV 205 GCYSTLDPIGQATVSLIVKSRG-GDVENMWGPVGSRTWQEHDVVSNPEGLRN---MAVYL 205 GCYSTLDPIGQTTVSLIVNSRG-GNVENMWGPTGSETWKAHDVTSNPEGLRD- - MAVYL 205 GCYSPVNTSGRELFNLAARVIG-GNPDLMWGRDITEQRRRNDVVANPSGIAS---MDTYI 205 GYLDTTSNGMPAAIGAALADAGGYNVNAMWGPAGSERWLENDPKRNVDQLR--G-KQVYV 203 GYLDTTSAGMPIAISAALADAGGYDANAMWGPVGSERWQENDPKSNVDKLK - -G-KTIYV 203 GYLDTTSIGMPAAIRAAOKDAGGYDSTAMWGPDGSODWIDHDPKLGVEALR - -G-ITTYV 201 GFLTTTTLGMPQAIQFAMRDAGGFDSAAMWGPPTSPEWEAHDPYLLADKLR--G-VSLYI 202 GYLNVSAPGMREALRVAMIDAGGYNIDAMAPPWG-POWLRMDPFVFAPRLKANN-TRLWI 235 GYLNISAPGMREAIRVAMLDAGGYNVDAMAPPWG-PQWLRMDPFVFAPNLIRNG-TRLWI 230 GYLNISAPGMREAIRIAMLDAGRFNVDSMAAPWS-PQWLRMDPFVFAPQLR--G-LPMYI 204 ALLDPSQGMGPSLIGLAMGDAGGYKAADMWGPSSDPAWERNDPTQQIPKLVANN-TRLWV 209 GLLDPSQAMGPTLIGLAMGDAGGYKASDMWGPKEDPAWQRNDPLLNVGKLIANN-TRVWV 209 GFLNP SEGWWPTLIGLAMNDSGGYNANSMWGP SSDPAWKRNDPMVQIPRLVANN-TRIWV 207 GYLTTTAPGMQTMLRLAMLDTGGFNVNAMYGSVINPRRFENDPFWNMGGLR - -G-KDVYV 209 GYLNTTAPGMQTLLRLAMLDTGGFNVNAMYGSIINPRRFENDPFWNMGGLA- -N-TDVYI 209 GYLSMSVPGTYLMMTLALQEVGGFNINNMYGSFFGLRRFQLDPLVNAAGLA- - G-KDVYV 206 
Nfa7210

Nfa 38260

Nfa32420

Nfa2 3770

Nfa 43800

Nfa 30260

Nfa 45560

Nfa2 5110

Nfa5610

$\mathrm{Ce} 0356$

Ncgl 0336

Dip0365

Ncgl2101

Ncgl0885

$\mathrm{Ce} 0984$

Ce1488

Ncgl0987

Dip2339

$\mathrm{Ce} 2709$

Ncgl2 777

Dip2193

Nfa1840

Nfa1810

Nfal 820

Nfa1830

1FOP

Rv3804C

Rv0129c

Ce2710

Ncgl 2779

Dip2194

Nfa7210

Nfa 38260

Nfa32420

Nfa2 3770

$\mathrm{Nfa} 43800$

Nfa 30260

Nfa 45560

Nfa2 5110

Nfa5610

Ce0356

Ncgl0336

Dip0365

Ncgl2101

Ncgl 0885

$\mathrm{Ce} 0984$

Ce1488

Ncgl0987

Dip2339

Ce2709

Ncgl 2777

Dip2193

Nfa1840

Nfa1810

Nfa1820

Nfa1830

$1 F 0 P$

Rv3804c

Rv0129c

Ce2710

Ncgl2779

Dip2194
STGNGIPGPYDTLN- - - - GPYALPGSYGLANQILIGGVIEAGTNYCTNNLKT- - RLDEL 257 STGTGLPGKWDTLN- - - - GPHAMPGSDGLTNQLVLGGILEAGADHCTRNMRD- -RLTQL 256 STGSGIPVLEDVQY - - - -YLNAAPGPMGAVN-LGLGVIIEAAVNQCTANLKN- -RLDSL 256 SSGSGLPGPHDTLA - - - - NPRLHNDDRQLLNQTLVGGAIESVTNLCTTRLAQ - -RTAEL 263 SSGSGLPGPLDNP - - - - - AAVGGDPMQLGYQLLFGAPLEAVTGMCTRQLRD - -RLQEL 253 TAGTGRPGALDSLQ - - - -GPGIDADPLALADQLLIGGALEAVAADCTSELGA - RLRAA 256 SAGDGRPGRHETLT - - - - APGIDGNPLDLVERTVVGGLMETVIGACTRPLVD- -RLTSL 253 SAASGIPGPIDRGG- - - -LPAPT- - - - - - - - LEAIARTCTAAFAD--RLAEL 238 STGTGIPGPHEAEL - - - - KPQLAEN- - - - - - IFLGGPVEVGVNICTVAFEQ - -RLRGL 246 SNASGLAGHWESANSPRFNGLDQAYLSLAMTETIVTGGLIEAATNKCTHDLKA--KLDHA 262 SNASGLAGEWESVDSPRFEGLNQQVQSIAMAETVVTGGIIEAATNKCTHDLKA--KLDSA 262 SNNSGAVGKYDLPSSPRLAGKDPVTIFATNLITATEGGIIEAGTNMCTHDLKV--KMDSL 260 FAGSGVFSELDVI - - - - - GDNAP IDEDAFKNRVLVGFEIEAMSNTCTHNLKA - - ATDQM 257 FAGSGVF SELDVI - - - - - GDNAP IDEDAFKNRVLVGFEIEAMSNTCTHNLKA - -ATDQM 257 FAASGVWSEVDVE--- - - GENAPEDEKGLKNRITVGFRIEALSNTCTHNLKA - - ATDYH 257 SAANGVVDEIDREE---- - - - -YADEPFYNLLAGTVLERGALSCTEALDDAMQD- -A 252 SAANGVVDDIDLAD--.-- - - SEKEP FYNLLAGVVLERGSLSCTEALDESMSR - -A 252 YVANGVATPSDVNG--.-- - - PKEDGPFTLFGNIVLEKMSYRCTQELEASVREKIA 254 SAGSGAD-DYGQDGSV----.---ATGPANAAGVGLELISRMTSQTFVD--AANGA 248 SSGNGAD-DFGKEGSV - - - - - - - AIGPANAAGVGLEVISRMTSQTFVD- - RASQA 248 SAGSGRD-DFGEPGSV------ - -ANKKGSYAGIGLEVISRMTTETFVA- -HARRA 246 SSGSGTTGPFDQASGI - - - - - - - PGVSTNYAGTGLEILSRLTSQNFVT--KLGEL 248

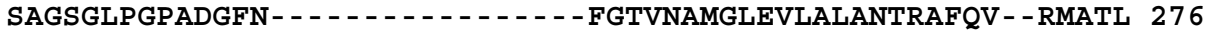
AAASGLPTSTDPPS - - - - - - - FNTLNGMGLEALALANTRAFQV - RMATL 271 SAASGLPGQHDRPNSP - - - - - - - -VGVFNTGNAMALEALSLVNTRAFQV - -RLKSL 250 YCGNGTPNELGGAN---.-- - - - - IPAEFLENFVRSSNLKFQD--AYNAA 247 YCGNGKPSDLGGNN- - - YCGNGTPSDLGGDN- - - - - - - - - - IPAKFLEGLTLRTNQTFRD- - TYAAD 245 SAASGLWGPQDNGTR - - - - - - - - VDHRINGSVLEAVSLATTRAWEA- - KARAE 252 SAASGLWSPQDDGVR - - - - - - - - VDHRLTGSVLEFVAMTSTRIWEA- -KARLQ 252

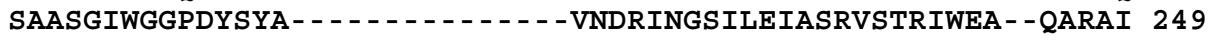

G-IPATYNFRPNGTHSWGYWNEEFPKSWPVLAKGL 291 G-IPATYDFQPRGTHSWGYWEDALKLSWPVLAKGL 290 G-IPATYEFTPVGTHYWPYWEQALHDSWPMLAEGM 290 GRTDITYNIRRPGTHSWGYWQDDLRDSWPMIARSI 298 R-IPATVDLRPTGTHAWGYWQEDLHKAWPMFEAAL 287 G-IPATVEVRPDGTHSWGYWEQDLRRCWPLFAAAL 290 A-VPATLALRP-GTHSWPYWQDDLHDSWPMFAAAI 286 G-IAAVHVDRPLGAHTWGQFETDLHESWPHLAAAL 272 G-IPARIDYSPVGTHSWSYWQDTLHASWSTIGRAL 280 GIP-ADWNLRPTGTHSWGWWQDDLRGSWDTFARSF 296 GIP-ADWNLRPTGTHSWGWWQDDLRGSWTTFARAF 296 NIP-ATFNFRNTGTHSWGYWEEDMVASWELFNMAF 294 GIDNINYDFRPTGTHAWDYWNEALHRFFPLMMQGF 292 GIDNINYDFRPTGTHAWDYWNEALHRFFPLMMQGF 292 GIDTIHYDFRPTGTHAWDYWNEALHRFFPLMMQGF 292 GMTHQVVDYKGAGAHNWRNFNEQLQPGWDAVKDAL 287 GMNHQVVDYKDSGTHNWRNFNPQLQPGWDAIKHAL 287 DPSRITFDYHDGGVHSWPYYRQQLPVAWANVSKGQ 289 G-VNVIANFRPSGVHAWPYWQFEMTQAWPYMADSL 282 G-VEVVASFRPSGVHSWEYWQFEMTQAFPHIANAL 282 G-VEVQAFFRPSGVHDWPYWQFEMTQAWPYMANAL 280 Q-IPATVNYRASGTHSWPYWDFEMRQSWPQAAAAL 282 GANNVTYDFPAVGVHNWRYWETEVYRMIPDLSANI 311 GGGNAVYSFPPFGIHAWNNWRDEAVRMMPDLSANI 306 G-IPAQFDFPATGTHSWKYWEGQLWNSRQGILDAL 284 GGHNAVFNFPPNGTHSWEYWGAQLNAMKGDLQSSL 282 GGHNGVFDFPDSGTHSWEYWGAQLNAMKPDLQRAL 282 GGRNGVFNFP PNGTHSWPYWNEQLVAMKADIQHVL 280 G-LNVTADYPNTGIHSWAQFSSQLHKTRDRVLNVM 286 G-LNPTADYPMYGIHGWAQFNSQLERTQGRVLDVM 286 G-LNLTTNYPLLGVHNWVQWRYQIEQSKPRILDVM 283 


\section{Bioinformation}

www.bioinformation.net

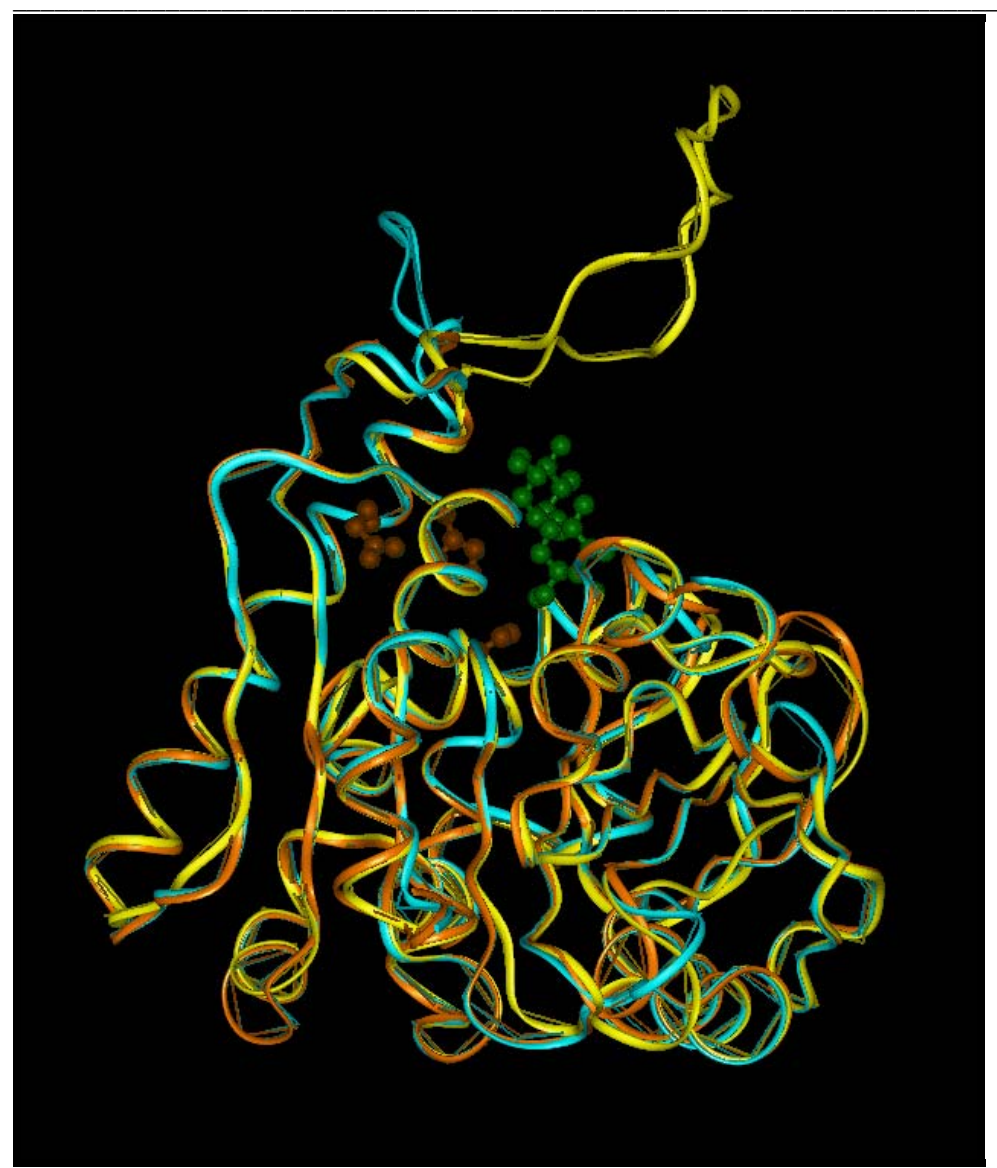

Figure 2: The structural superposition of representative CMN mycolyl-transferases (PDB Id: 1F0P (brown), Ncgl0336 (yellow), Ncgl0987 (blue). The side chains of the active site residues S126, E230, H262 (red) and trehalose 1151 (green) are represented in ball and stick model.

The three-dimensional models are useful to identify the positions of these highly conserved resides and regions of insertions. Further, we can also infer the nature of the substrate binding pockets defined by interactions with 'trehalose'. Evaluation of the three-dimensional models corresponding to corynomycolyl-transferases and nocardiomycolyl-transferases according to PROCHECK indicated more than $85 \%$ amino acid residues are in the allowed regions of the Ramachandran plot [29] suggesting that the models are of good quality. Further, according to Profiles-3D, the 'observed' scores for the models lie between 124-134 as 'expected', suggesting the compatibility of structure and sequence. Also, the RMSD of the respective structures is $\sim 0.68 \AA$ and residues that form the catalytic site S126, E230 and H262 can be highly superimposed. The conservation of catalytic residues and their positions in the three dimensional models indicated that all corynomycolyl transferases and nocardiomycolyl transferases must also retain catalytic activity. Examination of the models on computer graphics showed that, the conserved residues L39, P71, D81, W82, W97 and F100 constitute the 'hydrophobic tunnel'. These are needed in order to accommodate the alkyl chain of mycolic acid, indicating a functional conservation in these proteins. The invariant S126 and G260 are close to the catalytic active site comprising E230. The indole side chains of
W51 and W264 are perpendicular to each other and are in proximity to G124 associated with the $\beta 5$ strand. The amino acid residue D192 is away from the active site indicating that the conservation extends beyond the catalytic site in CMN mycolyl-transferases. We observed that the disulphide connectivity patterns are different. The structures of 1SFR (Ag85A) and 1F0N (Ag85B) consist a disulphide bridge connecting half-cystine residues on $\beta 5$ and $\beta 6$ strands. In some proteins, half-cystine residue on the $\alpha 10$ helix and halfcystine residue on the loop connecting $\beta 6$ strand and $\alpha 6$ helix are involved in the disulphide bridge. The information on the disulphide connectivity pattern is provided in Table 2 . Based on the structural superposition, we observed that the differences between these structures are only in the loop regions. The 27 amino acid residue insertion in Nfa1810 and Nfa1820 is located between the $\beta 5$ and $\beta 6$ strands that is away from the active site and we therefore predict that it may not be involved in the activity of the protein. According to the structure of 1F0P (Ag85B bound to the substrate trehalose), two substrate binding pockets are present. We observed that the variable region preceding the E230 forms an "insertion loop" close to the trehalose 1151 binding site 
Bioinformation www.bioinformation.net open access

\section{Hypothesis}

domain corresponding to the LGFP tandem repeats that may be responsible for maintaining the integrity of the cell wall. The presence of these LGFP repeats in C-terminal region of Nfa1840 and Dip2193 imply that these are also cell surface proteins and may be important in maintaining cell wall integrity in analogous manner.

\section{Conclusion:}

This work describes the comparison of the three-dimensional models for mycolyl-transferases in CMN genera. Although the sequence identities in some cases is as low as $17 \%$, yet the overall $\alpha / \beta$ fold characteristic of mycolyl-transferases is conserved. This conservation extends to the active site comprising amino acid residues; S126, E230 and H262. However, the amino acid residues comprising the substrate binding pockets defined by interactions with trehalose vary owing to certain mutations in some mycolyl-transferases. Also, significant differences are observed in the size of the substrate binding pocket owing to the close proximity of an insertion loop between the conserved W82 and W97. The size and nature of amino acid residues corresponding to the substrate binding pockets is likely to affect mycolyltransferase substrate specificity. These observations lead us to believe that during the course of evolution, gene duplication events followed by mutagenesis at the substrate binding pockets, may have resulted in those mycolyltransferases that are responsible for synthesis of polysaccharide-mycolate complex in an organism specific manner.

\section{Acknowledgement:}

HGR thanks UGC, New Delhi for a JRF fellowship. SA thanks CSIR New Delhi for a SRF fellowship. LGP thanks DBT, New Delhi for research funding. We thank referees for their valuable comments.

\section{References:}

[1] C. Cocito \& J. Delville, Eur J. Epidemiol., 1:202 (1985) [PMID: 2429862]

[2] M. D. Collins, et al., J. Gen Microbiol., 128:129 (1982) [PMID: 7086391]

[3] M. Daffé \& P. Draper, Adv Microb Physiol., 39:131 (1998) [PMID: 9328647]

[4] L. Alshamaony, et al., J. Gen Microbiol., 92:188 (1976) [PMID: 1107481]

[5] H. G. Wiker \& M. Harboe, Microbiol. Rev., 56:648 (1992) [PMID: 1480113]

[6] J. T. Belisle, et al., Science, 276:1420 (1997) [PMID: 9162010]

[7] C. Abou-Zeid, et al., Infect Immun., 56:3046 (1988) [PMID: 3141278]

[8] T. L. Ratliff, et al., J. Gen Microbiol., 134:1307 (1988) [PMID: 3143807]

[9] R. Ronning, et al., J. Biol. Chem., 279:36771 (2004) PMID: 15192106]

[10] H. Anderson, et al., J. Mol. Biol., 307:671 (2001) [PMID: 11254389] glycine, phenylalanine, proline) repeats in the C-terminal region of Ncgl2777 and Ce2709 [30] and suggested that the abnormal increase in the cell volume of C. glutamicum is due to the loss of C-terminal
168 


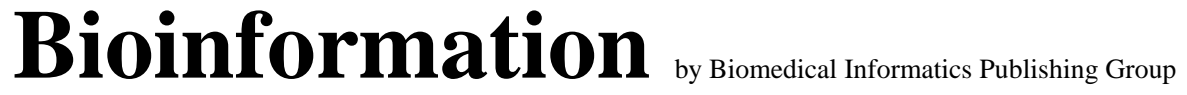

open access

\section{www.bioinformation.net}

\section{Hypothesis}

[11] R. Ronning, et al., Nat Struct Biol., 7:141 (2000) [PMID: [21] S. F. Altschul, et al., J. Mol. Biol., 215:403 (1990) 10655617]

[12] S. T. Cole, et al., Nature, 393:537 (1998) [PMID: 9634230]

[13] J. Kalinowski, et al., J. Biotechnol., 104:5 (2003) [PMID: 12948626]

[14] Y. Kawarabayasi, et al., Unpublished, (2002)

[15] A. M. Cerdeno-Tarraga, et al., Nucleic Acids Res., 31:6516 (2003) [PMID: 14602910]

[16] J. Ishikawa, et al., Proc Natl Acad Sci., 101:14925 (2004) [PMID: 15466710]

[17] S. Adindla, et al., Int J Biol Macromol., 34:181 (2004) [PMID: 15225990]

[18] S. Brand, et al., Arch Microbiol., 180:33 (2003) [PMID: 12740729]

[19] C. De Sousa-D’ Auria, et al., FEMS Microbiol Lett., 224:35 (2003) [PMID: 12855165]

[20] http://srs.ebi.ac.uk/ [PMID: 2231712]

[22] http://www.ncbi.nlm.nih.gov/BLAST/

[23] D. Higgins, et al., Nucleic Acids Res., 22:4673 (1994) [PMID: 7984417]

[24] A. Sali \& T. L. Blundell, J. Mol. Biol., 234:779 (1993) [PMID: 8254673]

[25] R. Sanchez \& A. Sali, Methods. Mol. Biol., 143:97 (2000) [PMID: 11084904]

[26] R. A. Laskowski, et al., J. Appl. Crystallogr., 26:283 (1993)

[27] R. Luthy, et al., Nature, 356:83 (1992) [PMID: 1538787]

[28] L. Kremer, et al., Lett Appl. Microbiol., 34:233 (2002) [PMID: 11940150 ]

[29] N. Ramachandran \& V. Sasisekharan, Adv Protein Chem., 23:283 (1968) [PMID: 4882249]

[30] S. Adindla, et al., Comp. Funct. Genomics, 5:2 (2004)

Edited by P. Kangueane

Citation: Ramulu et al., Bioinformation 1(5): 161-169 (2006)

License statement: This is an open-access article, which permits unrestricted use, distribution, and reproduction in any medium, for non-commercial purposes, provided the original author and source are credited. 